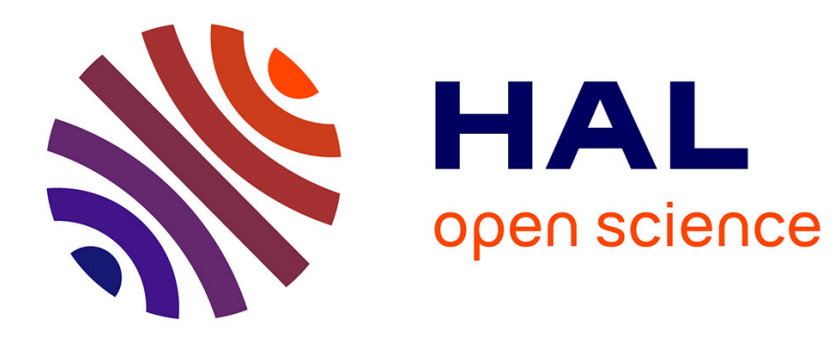

\title{
Consideration on fracture of solid-state materials
}

S. Yoshida

\section{- To cite this version:}

S. Yoshida. Consideration on fracture of solid-state materials. Physics Letters A, 2000, 270 (6), pp.320-325. hal-01092613

\section{HAL Id: hal-01092613 \\ https://hal.science/hal-01092613}

Submitted on 9 Dec 2014

HAL is a multi-disciplinary open access archive for the deposit and dissemination of scientific research documents, whether they are published or not. The documents may come from teaching and research institutions in France or abroad, or from public or private research centers.
L'archive ouverte pluridisciplinaire HAL, est destinée au dépôt et à la diffusion de documents scientifiques de niveau recherche, publiés ou non, émanant des établissements d'enseignement et de recherche français ou étrangers, des laboratoires publics ou privés. 


\title{
Consideration on fracture of solid-state materials
}

\author{
S. Yoshida
}

Physics Department, University of Florida, LIGO Livingston Observatory 19100 Ligo Lane, PO Box 940 Livingston, LA 70754 Phone:225-668-3130, E-mail: yoshida@phys.ufl.edu

\begin{abstract}
Fracture of solid state materials is considered based on a gauge theoretical approach. The critical condition in which a material is about to fracture is defined. This condition is analogous to the condition of electric breakdown of a gas. Supporting experimental results are presented.
\end{abstract}

In this letter, I will consider the fracture of solid state materials using a recent theory of plastic deformation (physical mesomechanics, PMM) [1]. Fol- 
lowing the mesomechanical interpretation that the fracture is the final stage of plastic deformation (PD), I will define the critical condition in which the material changes from the deforming phase into the fracturing phase. This newly defined condition (called the critical fracture condition, CFC) is different from the fracture criterion defined by PMM which I believe represents the post-fracture stage. Quite interestingly, however, the CFC is analogous to the condition in which a gas breaks down electrically, indicating analogy between the displacement field and the electromagnetic field. Below, I will describe the ground on which I have come up with the definition of CFC. I will present some experimental observations that support this definition. The analogy between the displacement field and electromagnetic field will also be discussed. Note that in this letter I will use the word fracture to mean that the material generates discontinuity (such as microckracks) and the word failure to mean that the material is broken into pieces.

Mathematically, PMM [1] describes PD as a GL(3, R) transformation of the local frame representing a deformation structural element (DSE). Requesting the local symmetry in the Lagrangian under this transformation, PMM has introduced a gauge filed and derived a Maxwell type field equation $[1,2]$. After summation over the group index, the field equation together with the Bianki identity can be written in the following form [1]:

$$
\begin{array}{r}
\operatorname{div} \vec{V}=J^{0} \\
\operatorname{rot} \vec{V}=\frac{\partial \vec{\omega}}{\partial t}
\end{array}
$$




$$
\begin{array}{r}
\operatorname{rot} \vec{\omega}=-\frac{1}{c_{t}^{2}} \frac{\partial \vec{V}}{\partial t}+\vec{J} \\
\operatorname{div} \vec{\omega}=0
\end{array}
$$

Here $\vec{V}(u, v)$ is the rate of the displacement, $\vec{\omega}$ is the angle of the local frame, $c_{t}$ is the phase velocity, $J^{0}$ and $\vec{J}$ are, respectively, the temporal and spatial components of the four vector representing the symmetry current.

$$
\begin{array}{r}
J^{0}=-g^{i j} \eta_{i}^{\alpha} \eta_{j}^{\beta} \\
\vec{J}=-g^{i j} \eta_{i}^{\alpha} D \eta_{j}^{\beta} C_{\alpha \beta}^{\mu \nu}
\end{array}
$$

where $g$ is the metric tensor, $\eta_{i}$ is the local frame, $D$ is the covariant derivative, $C_{\alpha \beta}^{\mu \nu}$ is the dimensionless elastic constant, and $\mu \nu \alpha \beta$ denote the external indices and $i$ and $j$ denote the internal indices. The solution of eq.(1) - (4) represent the wave character of $\vec{V}$ and $\vec{\omega}$. I will call them the $\vec{V}$ wave and $\vec{\omega}$ wave, respectively, and the plastic deformation wave (PDW), inclusively.

Material scientifically, PMM interprets PD as a continuous process of phase transition in which the material relaxes the stress in the most efficient fashion at each moment. The above-mentioned wave characteristic is explained as the self-organized wave due to the synergetic interaction between the translational and rotational modes of PD (translational-rotational interaction TRI), through which the material relaxes the stress energy. In this picture $J^{0}$ represents the source term associated with the stress concentration and $\vec{J}$ represents the current along the boundary of DSE associated with the rotation of the DSE as a whole [3]. 
A unique feature of PMM is its interpretation of the fracture as the final stage of PD [1]. As the PD progresses, the physical size of DSE increases. In the process approaching the fracturing stage, the PD shows the following pre-fracture criteria (PFC) [3, 4]. (i) the wavelength of PDW grows to be comparable to the sample size (wavelength PFC), (ii) the PDW becomes stationary (standing wave PFC), and (iii) a pair of mutually opposite vortices appear in the field of secondary temporal derivative of displacement (vortex PFC). We have observed wave characteristics of displacement and these PFC experimentally $[4,5,6]$. As the PD further progresses, the material becomes highly dissipative. This makes the TRI mechanism less efficient to occur, causing the PDW to decay. As a consequence, the material loses the mechanism of stress relaxation and tends to generate discontinuity as an alternative mechanism. This tendency enhances as the degree of PD increases. Eventually, the PD reaches the stage where the TRI mechanism stops functioning and the generation of discontinuity becomes the only possible mechanism of stress relaxation. PMM defines this stage as the fracture stage, and describes it by the following conditions called the fracture criterion [7].

$$
\begin{aligned}
& \frac{\partial \vec{\omega}}{\partial t}>0, \\
& \operatorname{rot} \vec{\omega}=0
\end{aligned}
$$

Let us consider the meaning of these conditions. Condition (7) indicates that when the material fractures the local frame rotates. From the wavelength $\mathrm{PFC}$, it can be said that the sample in this stage is represented by 
two DSEs and that the rotation of the local frame actually represents the rotation of these two parts of the sample. Fig. 1 illustrates this situation schematically. From the standing wave PFC, the boundary of this pair of DSEs stays at the same location on the sample until the fracture occurs. From the vortex PFC, the rotations of the two DSEs are opposite to each other. Based on these PFCs and considering that the fracture occurs when the TRI mechanism stops functioning, this rotational motion of DSE is most naturally interpreted as the situation where the rotation of DSEs induced by the preceding translational motion continues until the fracture takes place without being converted to the next phase of translational motion. Thus it can be said that the final stage of PD is characterized only by the rotational mode of displacement [3], and that eq. (7) represents such a rotation-only situation (rotation-only in the sense that the rotational motion is not converted to the next phase of translational motion).

On the other hand, in my opinion, condition (8) represents the postfracture stage. Mathematically, $\operatorname{rot} \vec{\omega}=0$ means that the angle of DSE is spatially uniform. To envision this situation, let us imagine a two dimensional model in which the DSE rotates in a x-y plane around the z-axis $\left(\omega_{x}=\omega_{y}=\right.$ 0 ). In such a model, condition (8) can be written as $\frac{\partial \omega_{z}}{\partial x}=\frac{\partial \omega_{z}}{\partial y}=0$, i.e., the angle of the local frame is constant along the $\mathrm{x}$ and $\mathrm{y}$ axes. This represents the situation where the whole sample is represented by a single frame, i.e., the frame can be defined globally. This can be interpreted as the situation where the gauge field as the connection field has disappeared because the stress has been removed by fracture. Apparently, this situation represents 
that the fracture is over, or the post-fracture stage. The meaning of eq.(8) as the condition representing the post-fracture stage will become clearer after I consider the CFC (critical fracture condition) below.

Now that (8) represents the post-fracture stage, there should be a condition that represents the stage where the TRI mechanism stops functioning. Let us call this situation the CFC. In my opinion, the CFC can be represented by $\frac{1}{c_{t}^{2}} \frac{\partial \vec{V}}{\partial t}=0$ for the following reasons.

First, the term $\frac{1}{c_{t}^{2}} \frac{\partial \vec{V}}{\partial t}$ represents the synergetic interaction between the translational and rotational displacement in eq.(3). Therefore, $\frac{1}{c_{t}^{2}} \frac{\partial \vec{V}}{\partial t}=0$ means that the TRI mechanism stops functioning.

Second, $\frac{\partial \vec{V}}{\partial t}=0$ indicates that the $\vec{V}$ wave loses its oscillatory character, i.e., it decays. This is consistent with the above-mentioned mesomechanical picture that toward fracture the material becomes dissipative and the PDW decays.

Third, $\frac{\partial \vec{V}}{\partial t}=0$ indicates stress relaxation. Since $\vec{V}$ has a physical unit of velocity (displacement in a unit time), $\frac{\partial \vec{V}}{\partial t}$ corresponds to acceleration, hence is proportional to a force. Therefore, it can be interpreted that $\frac{\partial \vec{V}}{\partial t}=0$ indicates that the stress is relaxed.

Forth, condition $\frac{1}{c_{t}^{2}} \frac{\partial \vec{V}}{\partial t}=0$ corresponds to the formation of an arc current in a gas discharge, indicating analogy between the mechanical fracture of a solid-state material and the electrical breakdown of a gas. Imagine that an electric field $\vec{E}$ is applied across a pair of electrodes placed in a gas and that you gradually increase $\vec{E}$. When $\vec{E}$ is low, the displacement current $\frac{\partial \epsilon \vec{E}}{\partial t}(\epsilon$ : the dielectric constant) is the dominant mechanism to lower the 
potential between the electrodes. As $\vec{E}$ increases, the ionization rate enhances and consequently the conduction current increases. Eventually, when the $\vec{E}$ reaches the breakdown threshold, the displacement current stops flowing, and instead, a large conduction current (i.e., an arc current) flows to bridge the electrodes. This is the moment when the gas breaks down.

This analogy with the electrical breakdown implies that the fracture of a solid-state material is also characterized by an initial stage where $\frac{1}{c_{t}^{2}} \frac{\partial \vec{V}}{\partial t}$ is decaying and the final (completion) stage where the current $\vec{J}$ becomes the dominant flow to relax the stress. Naturally, in this analogy $\frac{1}{c_{t}^{2}} \frac{\partial \vec{V}}{\partial t}$ corresponds to the displacement current and $\vec{J}$ corresponds to the conduction current. It is interesting to note that as the electric discharge enhances, the gas becomes more conductive, and this causes the electromagnetic wave to decay. This process is analogous to the situation where the PDW decays as the PD enhances. Also note that according to this consideration, the post-fracture condition (8) can be interpreted as corresponding to the post-breakdown stage where the arc current finishes flowing.

There are experimental observations that support these arguments. Previously, in a series of tensile experiments based on speckle interferometry, we observed wave characteristics in the transverse component of displacement $u$ (called the $\mathrm{u}$-wave). Here the transverse component is the one perpendicular to the tensile axis. All the u-waves observed rose when the stress-strain curve was about to reach the yield point, indicating that they certainly represent the PD. We have found that in some cases (depending on the initial stress condition and the type of material), the $\mathrm{u}$-wave shows a rather simple oscilla- 
tory characteristic [4] (called the simple u-wave). Fig. 2 shows two examples of such simple u-waves observed at several reference points along the tensile axis (see Fig. 3). While the waveform in the early stage is different from each other, both examples clearly show that (a) the u-wave decays (i.e., $\frac{\partial u}{\partial t}$ becomes zero) toward the end, and that (b) as soon as the u-wave decays the sample fails. (Note that the sharp rise observed at the very end of the figure represents that the two parts of the sample swing to a side after the failure.) All the samples that showed simple u-waves commonly show these features. The fact that the $\mathrm{u}$-wave decays toward the failure indicates that at least $\frac{\partial u}{\partial t}=0$ is a necessary condition of fracture, supporting the second argument mentioned above. Feature (b) can be interpreted as that when a simple $\mathrm{u}$-wave is observed the sample fails as soon as the CFC is satisfied for the first time. This is contrastive to the case when the sample does not show a simple $u$-wave. In that case, the early fractures do not lead to failure of the sample [4] (see below).

In these experiments, we also observed that when such a simple u-wave is about to decay, an optical band structure (called the white band, WB) $[5,8]$ begins to appear in the fringe pattern, and that the sample fails at the location of the WB [4]. Here the fringe pattern means the specklegram containing the interferometric fringe system from which we extract $u$ [9]. From the fact that the fringe system indicates that the sample rotates rather bodily above and below the WB, together with some other observations, we interpret that at this stage of PD the sample is characterized by two DSEs as shown in Fig. 1, and that the WB represents the current $\vec{J}$ flowing along 
the boundary of the DSEs associated with their rotations [10]. Fig. 4 shows a typical WB observed in a fringe system representing such bodily rotations. Fig. 2 shows the situation where the WB begins to appear when $\frac{\partial u}{\partial t}$ is about to decay. This observation supports the above mentioned picture that the fracturing process is initiated by decrease in the $\frac{1}{c_{t}^{2}} \frac{\partial \vec{V}}{\partial t}$ current and completed by the flow of current $\vec{J}$. Note that failure always takes place along the WB (i.e., along the path of current $\vec{J}$ ), and that this is analogous to the situation where the electrodes are bridged along the channel of the arc current in a gaseous breakdown.

In ref.[6] we observed that the appearance of a WB is accompanied by a sharp stress drop. Along with the above discussion, this can be interpreted as follows. As soon as $\frac{1}{c_{t}^{2}} \frac{\partial \vec{V}}{\partial t}$ becomes zero the stress is relaxed by the generation of discontinuity, and this is manifested as the sharp stress drop. At the same time, the material enters the rotation only-situation, and this is manifested as the appearance of the WB. This picture is consistent with the third argument mentioned above.

We have observed that when the initial stress concentration is relatively uniform, a number of WBs appear at various locations on the sample at different times [5]. In ref.[4], we observed that in this case, the u-wave does not show a simple decaying characteristic but rather a complicated oscillatory characteristic that continues until the sample fails. In ref.[6] we observed that every time such a WB appears at some location on the sample, the stress drops sharply as is the case of a simple u-wave. As the WB disappears from one location, however, the stress rises again. Thus the stress-strain 
curve shows a zigzag characteristic as WBs appear at new locations. While the analysis of this complicated u-wave is a subject of future investigation, one possible interpretation of these observations is as follows. When the CFC is satisfied, the stress is relaxed by the generation of discontinuity. Consequently, the stress drops sharply and the material enters into the rotationonly situation. However, since the level of discontinuity is not sufficient to lead the sample to failure, the material recovers from the rotation-only situation. Accordingly, the TRI mechanism resumes and a new u-wave is generated. This is why the observed u-wave does not show a simple decaying characteristic. At the same time, the work hardening resumes, causing the stress to rise again. After repeating this process, the material eventually undergoes the generation of discontinuity of fatal level, and fails.

It will be helpful to describe this transitional process from PD to fracture in a more intuitive picture. In such a picture, it can be described as follows. As the PD progresses the stress distribution becomes less uniform. This makes certain parts of the material weaker than the surrounding areas, and consequently, divides the whole sample into several, relatively rigid blocks, i.e., DSEs. In this situation, the motion associated with the PD can be represented by relative motions of those DSEs. As the PD further progresses, a particular part of the sample becomes weaker than the rest of the sample. Thus, at the final stage of PD, the sample can be represented by two DSEs, and a large bend-torsion moment operates in such a way that the sample bends about the boundary of these two DSEs. In this stage, the material still has a recovering force, and this causes the oscillatory motion observed 
in Fig. 2. Towards the fracture, this recoverability decreases, and eventually, the material loses the oscillatory motion. This is when the CFC is satisfied, and the two DSEs rotate in the same direction till the sample fractures.

It is interesting to consider the equivalent process in a gas discharge. As the voltage across the electrodes is increased, the ionization rate increases. Consequently, the charge density increases, and at the same time the charge distribution becomes less uniform. As the charge non-uniformity enhances, it becomes easier for the system to lower the potential by means of a large conduction current than the displacement current. Eventually, the displacement current stops flowing and an arc current flows to bridge the electrodes. It is well known that in a high pressure discharge, the arc formation can be suppressed by preionizing the gas so that the charge density becomes more uniform.

In summary, I have considered the transition from the final stage of PD to fracture. Based on the mesomechanical interpretation that a material fractures when it loses the TRI mechanism as a channel of stress relaxation, I have defined $\frac{1}{c_{t}^{2}} \frac{\partial \vec{V}}{\partial t}=0$ as the critical condition in which the material changes its phase from PD to fracture. For the case in which the first fracture leads to failure, I have presented some experimental results consistent with this interpretation. This critical condition is analogous to the condition in which an arc current flows in a discharge, breaking down the gas electrically.

Part of the experiment was made with the help of M. H. Pardede, B. 
Siahaan, S. Sijabat, H. Simangunsong, T. Simbolon. I greatly appreciate their help. I also thank S. Toyooka for his support and advice. 


\section{References}

[1] V. E. Panin in Physical Mesomechanics of heterogeneous media and Computer-aided Design of Materials, ed. V. E. Panin, Vol.1, (Cambridge International Science Publishing, (Cambridge, 1998).

[2] V. E. Egorshkin, Phisica, Izvestia, 33, 51 (1990) (Russian).

[3] V. E. Panin, private communication (1991)

[4] S. Yoshida et al. Phys. Lett. A, 251, 54 (1999)

[5] S. Yoshida et al. Optics Express, 2, 516 (1998).

[6] S. Yoshida et al. Theor. and Appl. Fract. Mechanics, 27, 85 (1997).

[7] See p.28 of ref. [1].

[8] Sanichiro Yoshida et al. Jpn. J. Appl. Phys. 35, L854 (1996).

[9] S. Yoshida et al. Appl. Opt. 36, 266 (1997).

[10] S. Yoshida, J. Phys. Mesomech. 2, 5 (1999) (Russian). 
Figure 1: A pair of DSEs in the pre-fracture stage. The local frames representing DSE1 and DSE2 rotate in mutually opposite directions. The rotations are exaggerated and the gap between the DSEs does not indicate that the sample is fractured.

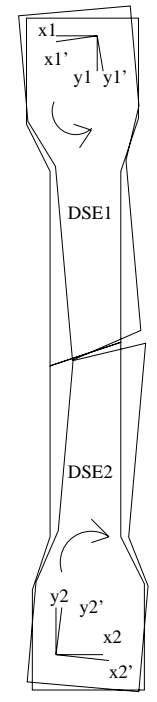


Figure 2: Examples of simple u-waves. line 100, 250, 400 denote three reference points on the tensile axis at which displacement $u$ was extracted. An arrow indicates when the WB begins to appear.
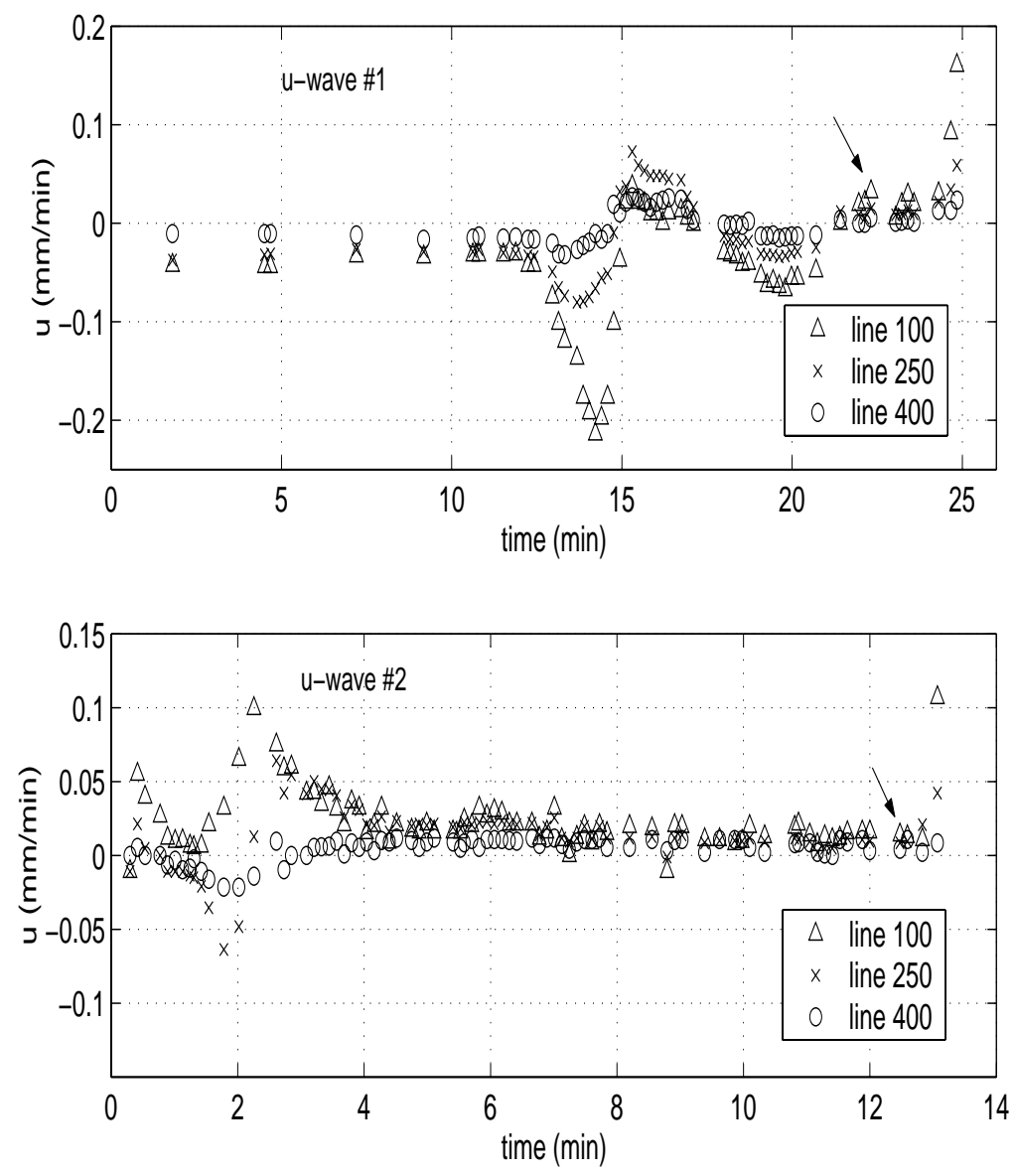
Figure 3: Reference points at which displacement is extracted.

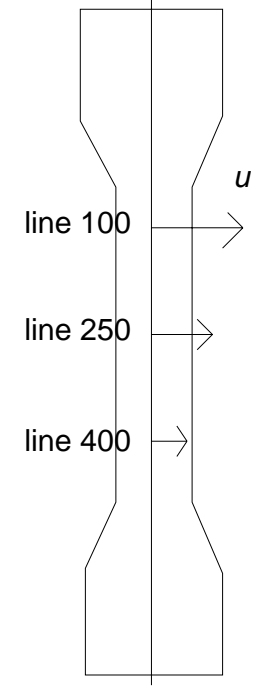

Figure 4: An example of WB. The horizontally-parallel, quasi-equidistant fringe systems above and below the WB represent bodily rotations of the respective parts of the sample.

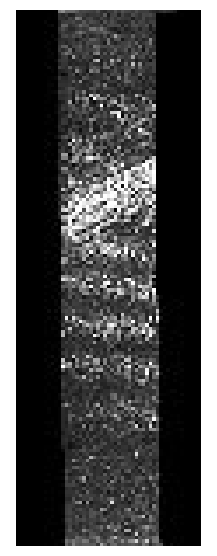

